\title{
PROPUESTAS CURRICULARES PARA RESPONDER A LA DIVERSIDAD DEL ALUMNADO DE PRIMARIA DESDE CONTEXTOS INCLUSIVOS
}

\author{
Ma Asunción Jiménez Trens \\ Universidad de La Rioja
}

\section{RESUMEN}

Este estudio parte de una serie de argumentos que ponen de manifiesto la necesidad de un modelo de inclusión educativa como factor de indudable calidad en la educación. Modelo que encuentra su sentido, justamente, en hacer posible una respuesta educativa adaptada a la diversidad del heterogéneo alumnado que integra los centros y aulas escolares. Trata, por tanto, de suscitar la reflexión y el debate sobre variadas oportunidades que desde un marco curricular común pueden ofrecerse a la totalidad del alumnado y que permiten individualizar la enseñanza y personalizar la educación. Teniendo en cuenta tales consideraciones se presentan propuestas que, sin pretender ser exhaustivas, ofrecen algunas pautas que pueden tomarse en consideración para el diseño y desarrollo del currículo en el centro: Proyecto Educativo, Proyecto Curricular y Programación de Aula.

PALABRAS CLAVE: Inclusión educativa, respuesta educativa a la diversidad, currículo, educación primaria.

\begin{abstract}
This study set off a serial of arguments that show the necessity of a model of educative inclusion as a doubtless quality factor in the system education. A model that finds its sense just in achieve an educative answer fitted with the diversity of the heterogeneous students who integrate the schools and the classrooms. This model tries, therefore, to provoke a reflection and to debate about how inside common curricular environment different opportunities can be offered to all the students, allowing an individual and personalized education. Taking these considerations into account and with no intention of being exhaustive, some proposals are presented as guidelines for both the design and development of the school curriculum: Educative Project, Curricular Project and Classroom Planning.
\end{abstract}

KEY WORDS: Educative inclusion, educative answer to diversity, curriculum, primary school. 


\section{INTRODUCCIÓN: RELEVANCIA DEL MODELO DE INCLUSIÓN EDUCATIVA}

Los contextos de inclusión educativa representan, en mi opinión, el marco adecuado y necesario para llevar a cabo la planificación y actuación educativa adaptada a las necesidades de todo el alumnado. Tienen su fundamento en el modelo de inclusión educativa (Ainscow, 2001 a y 2001 b; Arnaiz, 2003; Booth y Ainscow, 2000; Cardona, 2005; Gairín, 2001; Parrilla, 2002; Susinos, 2002 y 2005) que persigue, entre otros, los fines siguientes: a) que TODOS los miembros del centro escolar se sientan partícipes del mismo, aceptados, seguros y bienvenidos; b) aumentar la participación de todos para que logren el máximo aprendizaje y desarrollo personal y social, lo que implica, entre otras cosas, identificar las barreras que la impiden o dificultan presentes en la organización del centro y del currículo así como asumir el compromiso de su eliminación progresiva; c) dedicar una atención especial a grupos e individuos con mayor riesgo de exclusión escolar y social; d) crear culturas inclusivas en cada centro escolar, en el sentido de construir una comunidad escolar segu$\mathrm{ra}$, acogedora, estimulante y colaboradora en la que cada docente es considerado como el fundamento primordial para que todo el alumnado alcance mayores niveles de logro; e) elaborar políticas inclusivas que tiene que ver con la línea estratégica que cada centro decide, planifica y organiza para regular la vida del centro y aulas con el objetivo de que mejore el aprendizaje y la participación de todos los componentes; f) desarrollar prácticas inclusivas cotidianas tanto en actividades formales de aula como en las extraescolares; g) fomentar las mutuas relaciones entre escuela y sociedad con el fin de movilizar todos los recursos del centro y de la comunidad local para conseguir el aprendizaje de todos y el camino hacia una sociedad cada vez más inclusiva. Si bien es cierto que este modelo tiene su origen en el ámbito de la Educación Especial en los países anglosajones, fue ratificado por una mayoría de representantes de la política educativa a nivel mundial en la Declaración de Salamanca (UNESCO,/MEC, 1994; Echeita y Verdugo, 2004; Jiménez Trens, 2004; Saleh, 2004) con una clara apuesta por abarcar a todos los integrantes de la comunidad educativa, trascendiendo el universo de las necesidades educativas especiales asociadas a discapacidad.

Una escuela de esta naturaleza, en consecuencia, es una escuela eficaz. Es así porque persigue que todo su alumnado mejore su rendimiento identificando con precisión las dificultades que puede experimentar cualquier estudiante en su aprendizaje, sin necesidad de un etiquetado previo, y proponiendo formas de actuación adaptadas para superarlas (Díaz Allué y otros, 1997; Díaz Allué, 1999; García García, 2002 y 2005). Al mismo tiempo, se tiene en cuenta a todo el alumnado al que se considera diferente por naturaleza; aún más, esa misma diversidad se interpreta como oportunidad de enriquecimiento de los grupos a los que pertenece. Representa, por tanto, una clara posibilidad de lograr una EDUCACIÓN de CALIDAD para TODOS.

Conseguir que este modelo se haga realidad ha de responder, por tanto, a una preocupación nuclear de TODO EL PROFESORADO, trabajando en colaboración, sin olvidar que también compete a la comunidad educativa en su conjunto.

Además, en lo que se refiere a los docentes, conviene resaltar que en su trabajo se ve implicada y comprometida su personalidad total. Así, sus decisiones y actuaciones profesionales están "teñidas" y marcadas por sus valores, reflexiones, sentimientos, emociones, creencias y teorías personales que, seguramente, son las que le 
van a proporcionar la motivación, el calor, la fuerza motriz para actuar y, en definitiva, pueden cristalizar en un compromiso. Un compromiso personal y profesional, fuerte y sostenido, que se revela como imprescindible para abordar el reto de la respuesta a la diversidad y que, aún siendo consecuencia de la reflexión personal y de la autonomía, puede ser más llevadero cuando se generan sentimientos y emociones de genuino afecto hacia el alumnado y de seguridad en la profesión. Así, la primera forma de respuesta a la diversidad se produce en lo que podríamos denominar el "laboratorio mental o personal" del docente. Tan importante puede ser la persona del docente que Riart Vendrell (2007: 31), en mi opinión muy acertadamente, considera que: Un colectivo de docentes y tutores "sanos" no estresados, en plenas facultades y con máxima capacidad es el primer elemento a preservar en lo que se refiere a la atención a la diversidad.

Además, es preciso crear un clima de centro que haga posible esa adecuada respuesta a todos. Algunos rasgos que pueden contribuir a la creación de ese ambiente, sobre la base de las propuestas de Booth y Ainscow (2000), son los siguientes: a) considerar todas las manifestaciones de la diversidad como objeto de atención debida, no limitarlas únicamente a las deficiencias o a las minorías étnicas; b) interpretar la presencia de alumnos que necesitan más ayuda para aprender, como un reto para la mejora de la docencia y no como un impedimento; c) asumir la variedad de lenguas, etnias, costumbres, capacidades, etc. como oportunidades de enriquecimiento para todos; d) reconocer el valor inherente a todo ser humano; e) interpretar las dificultades de aprendizaje como consecuencia de la interacción entre los sujetos y el contexto escolar, fomentando las formas de apoyo ordinarias, para todos los estudiantes, y utilizando aquellas extraordinarias como complemento o alternativa cuando las primeras resulten insuficientes.

En el plano legislativo la Ley Orgánica de Educación (LOE, 2006) parece apuntar a una respuesta a la diversidad con una orientación inclusiva. Se observa, sobre todo, en la exposición de sus fundamentos (Preámbulo), en el establecimiento de la atención a la diversidad como principio fundamental que debe regir la enseñanza básica, así como en el enunciado de la finalidad de las enseñanzas mínimas (Título Preliminar), también cuando trata de garantizar la equidad en la educación a partir del principio de inclusión (Título II) y en el reconocimiento de la autonomía pedagógica, de organización y de gestión de los centros (Título V, capítulo II).

Teniendo en cuenta las anteriores consideraciones se presentan, a continuación, algunas propuestas curriculares que desde contextos inclusivos permiten una respuesta educativa adaptada a la diversidad del heterogéneo alumnado que integra los centros y aulas escolares. Se trata, por tanto, de reflexionar sobre variadas oportunidades que desde un marco curricular común pueden ofrecerse a la totalidad del alumnado y que permiten individualizar la enseñanza y personalizar la educación.

\section{RESPUESTA CURRICULAR PARA TODO EL ALUMNADO}

La normativa legal emanada de las correspondientes administraciones educativas establece, además de otras cuestiones, las bases para el diseño del currículo. Ese diseño es el que va a permitir la organización de la respuesta a la diversidad en todo el país, en cada comunidad autónoma y en cada centro. Según como sea elaborado se dispondrá de distintas posibilidades para conseguirlo desde un enfoque tendente hacia la inclusión o la exclusión. Cuando el currículo legal propicia la adaptación a 
los centros, aulas y estudiantes se le suele considerar como una potente vía de respuesta a la diversidad desde un marco inclusivo; esto ha llevado a algún autor a afirmar que la vía general y más importante de atención a la diversidad se deriva del propio carácter abierto y flexible del currículo (Soler, 1999:7). Flexibilidad que reclama la autonomía de los centros para poder ejercerla, así como una adecuada formación del profesorado para saber afrontar el reto que representa la respuesta creativa a las demandas educativas.

En consecuencia, la planificación del currículo para todos los estudiantes sigue un orden progresivo: en primer lugar, la administración educativa central diseña el currículo que establece las enseñanzas mínimas de cada etapa regulado por real decreto, así como las administraciones autonómicas emiten su correspondiente decreto que configura el currículo para su comunidad autónoma; a continuación, desde cada centro, a partir del estudio y valoración crítica de los anteriores documentos, se procede a adaptar, desarrollar, "recrear" su currículo mediante los proyectos educativo y curricular; por último, se confeccionan las programaciones de aula que permitirán acercar y concretar tales propuestas a las necesidades de cada grupo-aula.

\section{ENSEÑANZAS MÍNIMAS DE LA EDUCACIÓN PRIMARIA}

La legislación regula los aspectos básicos del currículo, correspondiendo éstos a una estructura de enseñanzas mínimas. En coherencia con la Ley Orgánica de Educación (2006), la norma legal relativa al currículo de esta etapa es el $R$. D. 1513/2006, de 7 de diciembre, por el que se establecen las enseñanzas mínimas de la Educación primaria. Analizado el real decreto a la luz del tema que nos ocupa, merece destacarse los siguientes aspectos. En primer lugar, la prescripción legal de que los centros docentes deben desarrollar y completar el currículo establecido por las administraciones educativas, representa una clara oportunidad de adaptar el currículo oficial a las necesidades educativas del alumnado de cada centro y eso, en sí, ya es un modo muy importante de responder a la diversidad. En segundo lugar, que la respuesta a un alumnado diverso se constituye en principio básico de la intervención educativa tratando de garantizar el desarrollo de todos así como su atención personalizada; en consecuencia, se entiende la diversidad como manifestación de distintas formas de aprender y de distintas características que condicionan el propio aprendizaje. Ello significa, desde mi punto de vista, una concepción inclusiva de la diversidad. Visión ratificada, así mismo, en el planteamiento de las medidas de atención a la diversidad como garantía de una educación de calidad para todos los alumnos y alumnas, para lograr su éxito y responder a las distintas necesidades. En tercer lugar, la introducción por primera vez de las competencias entre los elementos básicos del currículo: constituyen una oportunidad de integrar y aplicar variados aprendizajes al mismo tiempo que permiten amplia variedad en la forma de llegar a su dominio y con un grado diferente de desarrollo; y eso es coherente con una respuesta adaptada a la diversidad desde un planteamiento de inclusión. En cuarto lugar, se explicita que la "acción educativa en esta etapa procurará la integración de las distintas experiencias y aprendizajes del alumnado y se adaptará a sus ritmos de aprendizaje" (Art. 4.7). Precisamente, ésta es una de las demandas del modelo inclusivo para intentar que todo el alumnado aprenda al máximo de sus capacidades y esfuerzo, reconociendo diversas fuentes de aprendizaje; al mismo tiempo, la necesidad de 
que desde la escuela se le ayude a integrar y dotar de sentido a las distintas experiencias vitales y escolares de cada uno y formar estructuras mentales que faciliten la integración del saber y la progresión en su dominio. En quinto lugar, la importancia otorgada a las funciones de la evaluación. Destaca el carácter formativo y orientador derivado de la función de diagnóstico que permite averiguar cómo son los procesos de aprendizaje de cada alumno y así poder aplicar de modo inmediato las medidas de mejora pertinentes; así mismo, la práctica de la evaluación continua con finalidad formativa que permite adoptar medidas de refuerzo educativo en cualquier momento del ciclo. También es verdad que cumplir con propiedad estas funciones requerirán potenciar la figura de los orientadores en los centros de primaria y las funciones de tutoría de los maestros mucho más de lo que ha sido hasta el momento.

\section{CURRíCULO EN EL CENTRO EDUCATIVO}

Tomando como base el currículo básico, cada centro ha de adaptarlo a su contexto geográfico, sociocultural y educativo. Dos principios son esenciales para que este proceso cumpla su finalidad. Uno es el de autonomía de los centros. Otro es el de atención a la diversidad. Ambos tienen cabida en un sistema educativo flexible que encuentra su razón de ser, precisamente, en organizar y propiciar una educación de calidad para todos. Los principales documentos de centro, de carácter general, en los que se plasman las decisiones curriculares que permiten atender a la diversidad son el proyecto educativo, el proyecto curricular y las programaciones de aula. No obstante, no hemos de olvidar la necesidad de complementar esta planificación curricular con otras medidas de carácter específico, que deberán abordarse oportunamente.

\subsection{PROYECTO EDUCATIVO DE CENTRO}

El proyecto educativo de centro (PEC) desempeña un papel fundamental en la respuesta a la diversidad. Es el instrumento mediante el que toda la comunidad educativa reflexiona, debate, negocia, acuerda y decide un modelo educativo válido para el centro. De alguna manera se puede afirmar que significa la ocasión de conjugar los ideales, deseos e ilusiones con la realidad y la praxis. En él, a partir del análisis de la realidad del centro y de su entorno sociocultural y familiar que permitirá el diagnóstico preciso de las necesidades de aprendizaje y de participación, se han de decidir los rasgos de identidad pedagógica y los objetivos de formación para toda la comunidad educativa. Así mismo, y de modo coherente con lo anterior, se ha de concretar la estructura organizativa del centro, estableciendo las funciones de cada uno de los componentes organizativos y las relaciones entre sí y con los demás sectores sociales. A continuación, se presentan algunas propuestas para la elaboración del PEC con la intención de responder a la diversidad desde un modelo inclusivo.

En primer lugar, es conveniente que el profesorado reflexione, explicite y contraste sus ideas acerca de una serie de cuestiones, entre otras, las siguientes: qué significa el modelo de educación inclusiva y que implicaciones representa, qué entiende por diversidad, a qué alumnado se refiere, qué finalidades persigue la atención a la diversidad, qué significado atribuyen a las diferencias individuales. Esta reflexión compartida puede servir para que el claustro se haga consciente de hacia qué modelo se decanta su interpretación: si es hacia uno de corte selectivo o hacia otro de carácter más integrador/inclusivo. Conviene señalar que, en caso de que se detecta- 
ra una orientación selectiva, sería conveniente instaurar acciones dirigidas a modificar paulatinamente tales concepciones en el sentido de propiciar la asunción del modelo inclusivo. Entre esas acciones cabe mencionar la formación permanente del profesorado y la organización del apoyo que puedan precisar los docentes para que se sientan capaces de afrontar la diversidad de las aulas. Formación y apoyo que se revelan más eficaces cuando se llevan a cabo en el propio centro, bien sea mediante la presencia de asesores externos o internos que faciliten tal avance, o por los medios que se estimen convenientes.

En segundo lugar, es preciso que en el PEC se plasmen acuerdos acerca de cómo proceder para identificar y evaluar las necesidades educativas especiales, las barreras para el aprendizaje y la participación que pueden experimentar grupos o individuos en situación de riesgo o de los que se sospeche su existencia. Así mismo, esta evaluación psicopedagógica ha de permitir la identificación funcional de las necesidades educativas en las diferentes áreas y ámbitos de la personalidad para poder determinar con precisión las ayudas pedagógicas, refuerzos o medidas que necesite cada sujeto. En cualquier caso, se recomienda destacar las capacidades y aquello que pueden conseguir más que las limitaciones o deficiencias; en consecuencia, la ayuda educativa será estimulante y tratará de lograr el desarrollo de todas las potencialidades del ser humano.

En tercer lugar, al conocer con precisión y tener en cuenta las características de todo el alumnado se pueden planificar las diversas decisiones organizativas buscando la mayor eficacia para el aprendizaje de todos y para fomentar la convivencia enriquecedora y su participación. La práctica de este modelo educativo requiere una organización flexible del centro y del aula, tanto en lo relativo al trabajo docente como al del alumnado. Además, se considera premisa básica de una organización de esta naturaleza, la comunicación, coordinación y colaboración entre todos los docentes y de éstos con los demás profesionales de la educación tales como Orientadores, Profesorado de Apoyo, etc.; sería imposible responder adecuadamente a las necesidades del alumnado si cada docente actuara de modo aislado. Alguna de las estrategias organizativas (Blanchard y Muzás, 2005;Oliver, 2003) que pretenden ofrecer respuesta a las múltiples y variadas necesidades educativas del alumnado, en coherencia con el modelo propuesto, son las siguientes: A) agrupamiento flexible del alumnado: representa una de las soluciones más adecuadas para garantizar la inclusión y la eficacia en la enseñanza y el aprendizaje de todos. Combina la riqueza que representa el grupo heterogéneo en género, intereses, ritmo de trabajo, capacidades, valores culturales, etc. con la eficacia de los agrupamientos homogéneos temporales, siempre que se cumplan una serie de criterios relativos a la forma de hacer los agrupamientos, a la coordinación y trabajo en equipo de los docentes y a la metodología de enseñanza/aprendizaje adaptada, así como a los criterios de evaluación y de flexibilización, y la información y comunicación al alumnado y familias. B) Organización de grupos en desdobles: el aula queda dividida en dos grupos heterogéneos, con lo que se mantiene la riqueza de la diversidad y se reduce la complejidad al disminuir el número de alumnos a los que se ha de atender. C) Organización del trabajo docente de tal modo que tanto el profesorado de apoyo como el regular sean y "se sientan" responsables de todos los alumnos. D) Los "Grupos de Apoyo entre Profesores" (GAEP) (Parrilla y Gallego, 1999) se revelan como un fecundo modelo de apoyo colaborativo entre profesores, que representan una alternativa a la concepción del apoyo como práctica terapéutica profesional ais- 
lada. E) En cuanto a la organización de espacios, es preciso tener en cuenta una serie de criterios para promover el aprendizaje y la participación de todos y, en general, para que permitan la realización de los fines que persigue el modelo inclusivo, expuestos anteriormente. En cualquier caso, debe facilitar la realización de trabajo autónomo por parte del alumnado, así como la interacción entre iguales, el aprendizaje cooperativo y, en general, su implicación activa en el aprendizaje; así mismo ha de propiciar el trabajo colegiado de los docentes. Tampoco debe olvidarse que las decisiones acerca de cómo ubicar a los alumnos y colocar las mesas en el aula son neutras; indican concepciones acerca de cómo se interpretan las diferentes prácticas y ofrecen o disminuyen posibilidades para el aprendizaje $y$, de igual modo, representan para el alumnado mensajes no verbales pero muy expresivos de donde colocan a determinados compañeros (disruptivos, más capaces, con discapacidad, inmigrantes, etc.). Es conveniente, también, abordar con imaginación el aprovechamiento de todo tipo de espacios, no sólo las aulas, como escenarios de aprendizaje de todos. Así mismo, no debe haber barreras arquitectónicas ni de cualquier otra naturaleza que impidan o disminuyan la plena integración y participación de todas aqueIlas personas que presentan alguna discapacidad motora o sensorial. Es más, el espacio ha de emitir mensajes de acogida para que todos los componentes de la comunidad educativa se sientan partícipes, aceptados y seguros.

Por último, insistir, una vez más, en el reconocimiento de que un proyecto de esta naturaleza sólo será posible si la comunidad educativa se plantea, a partir de sus prácticas presentes, modificar paulatinamente todos aquellos aspectos, que se evalúen como susceptibles de mejora, relativos a la creación de culturas inclusivas (construyendo comunidad y estableciendo valores inclusivos), a la elaboración de políticas (haciendo una escuela para todos y organizando el apoyo para atender a la diversidad) y al desarrollo de prácticas inclusivas (Booth y Ainscow, 2002).

\subsection{PROYECTO CURRICULAR DE CENTRO}

Este documento también desempeña un papel clave en la respuesta a la diversidad de todo el alumnado del centro. Hay dos razones que avalan esta afirmación. Una, porque cuanto más se tenga presente la diversidad del alumnado desde los primeros niveles de planificación curricular, menos necesarias serán las medidas específicas de diseño e intervención para alumnos en riesgo de exclusión, al mismo tiempo que puede ejercer un efecto preventivo del fracaso escolar en numerosos alumnos y alumnas. La otra, porque es un instrumento fundamental para la gestión de la autonomía curricular en los centros. Y esta autonomía va a permitir al profesorado adoptar las decisiones adecuadas para desarrollar una enseñanza ajustada a las diversas necesidades de los estudiantes. Mediante el proyecto curricular, por tanto, el centro desarrolla y completa el currículo establecido por las administraciones educativas. Así mismo, y junto al PEC, pretende ser un instrumento válido para dar respuesta a las características y a la realidad educativa de cada centro.

Diseñar el proyecto curricular de centro (PCC) implica un proceso de toma de decisiones relativas a las intenciones educativas (explicitar tales intenciones significa decidir de modo concreto qué se considera necesario enseñar y aprender), a la organización y secuenciación de contenidos, a la metodología didáctica y a la evaluación. Este proceso, para que sea eficaz, ha de ser consecuencia de una reflexión conjunta y coordinada del profesorado con intención de planificar, aplicar y evaluar el currículo apropiado a todos y cada uno de los estudiantes. Para ello, partiendo del 
proyecto educativo inclusivo establecido, habrá de tenerse presente, entre otros aspectos, la normativa legal que lo regula y la realidad del centro. A continuación, se presentan algunas consideraciones que pueden tenerse en cuenta en la confección del proyecto curricular para responder a la diversidad.

En primer lugar, se ha de contextualizar el currículo que se va a crear en el centro. Para ello se pueden plantear una serie de cuestiones, tales como las siguientes: ¿cuáles son las principales características de nuestros alumnos y alumnas? ¿cómo son sus formas de aprender? ¿qué necesidades de aprendizaje y de participación tienen? ¿qué posibilidades y capacidades relevantes presentan? ¿tienen alguna característica significativa que influya en las demás?; la caracterización que hacemos de nuestros alumnos y alumnas ¿es aplicable en términos generales a todos ellos o existen diferencias sustanciales y apreciables entre algunos grupos? ¿hay algún grupo o individuo que por su capacidad, origen sociocultural, etnia, o por cualquier otra razón esté en riesgo de exclusión? Mediante la respuesta a estas cuestiones y otras similares podemos disponer del perfil de nuestros alumnos y alumnas y de sus circunstancias familiares, socioculturales y educativas, así como de la presencia de algún grupo o sujeto con especial relevancia, que hay que tener en cuenta para elaborar un currículo adaptado al centro real.

En segundo lugar, el diseño de competencias básicas (comunicación lingüística, matemática, conocimiento e interacción con el mundo físico, tratamiento de la información y competencia digital, competencia social y ciudadana, cultural y artística, competencia para aprender a aprender, autonomía e iniciativa personal) en el real decreto de enseñanzas mínimas de Educación Primaria (2006) representa una buena oportunidad de planificar la enseñanza y el aprendizaje de modo ajustado a las necesidades y características detectadas en el centro. Así mismo, permite organizar el aprendizaje de modo integrado en torno a lo que se ha considerado los "pilares de la educación": saber, saber hacer, saber ser y saber convivir con una clara orientación hacia la aplicación en variadas situaciones. La finalidad que persiguen es que todos los estudiantes alcancen, mediante la práctica de un aprendizaje permanente extendido a toda la vida, su plena realización personal y social. Al explicitar saberes imprescindibles que deben iniciarse en Educación Infantil y Primaria y desarrollarse al término de la educación obligatoria, van a actuar como claros referentes en el proceso de enseñanza/aprendizaje en todas sus fases: planificación, realización y evaluación; de este modo, se puede organizar la enseñanza para atender a las distintas capacidades e intereses del alumnado con distinto grado de perfección en su logro. Así mismo, en el PCC hay que decidir cómo se va a trabajar el desarrollo de las competencias en cada área y ciclo, ya que no existe una relación unívoca área-competencia: cada área contribuye al desarrollo de varias competencias y, a su vez, cada competencia se consigue como consecuencia del trabajo en varias áreas. Por tanto, cabe plantearse algunas cuestiones cuya respuesta puede guiar la confección del PCC a este respecto; pueden ser las siguientes: ¿qué aspectos de cada una de las competencias resultan más adecuados al perfil del alumnado de nuestro centro? ¿cómo va a contribuir cada una de las áreas que configuran el currículo básico al desarrollo de todas las competencias? ¿cuáles son los diferentes grados de perfección en el logro de las competencias que se puede establecer atendiendo al perfil de nuestro alumnado y a lo que es imprescindible en cada una de las competencias? ¿cómo concretar en objetivos y contenidos en las diferentes áreas el inicio y progresivo desarrollo de cada una de las competencias? etc. 
En tercer lugar, se puede decidir si se va a priorizar o reforzar algunas capacidades que en determinados alumnos se presentan poco o nada desarrolladas, con el fin de garantizar el desarrollo de todas las competencias.

En cuarto lugar, conviene contextualizar las experiencias de enseñanza y aprendizaje, que vayan a llevarse a cabo en el centro, en el entorno socio-natural y cultural próximo con el fin de dotar de la máxima significación vital, social, psicológica y educativa la consecución de objetivos y competencias. Como los intereses y motivaciones del alumnado pueden ser muy diversos, habrá que considerar contextos muy variados para que sus intereses y expectativas encuentren eco en el currículo del centro (por ejemplo: medio rural/urbano, patrimonio natural e histórico-artístico local, fiestas, tradiciones y eventos culturales propios, etc.).

En quinto lugar, en relación a los contenidos, es importante: A) Organizar, secuenciar y distribuir. Para ello hay que tener en cuenta la dificultad que presentan y así hacer una ordenación según su grado de dificultad por ciclos y cursos, de tal modo que pueda producirse un aprendizaje escalonado. No obstante, también se suele recomendar organizarlos mediante una secuenciación progresiva que interrelacione los contenidos de una o de varias áreas y que siga un avance en espiral. Ello con la intención de buscar cada vez mayor complejidad, profundidad, amplitud y detalle, o con menos ayudas, que permitan realizar un proceso personal de asimilación y construcción del saber. Así mismo, en esta organización de contenidos, se considera conveniente poner el énfasis en aquellas competencias comunes a varias o a todas las áreas. B) Fijar prioridades, establecer un equilibrio adecuado entre los distintos tipos de contenidos de todas las áreas y agruparlos en los casos que se considere conveniente. C) Con el fin de contextualizar los contenidos en el centro, cabe matizar, comentar alguno de los objetivos y contenidos, orientarlos de acuerdo al perfil del centro, así como plantearse incorporar aquellos que se consideren necesarios.

En sexto lugar, cuando el equipo docente decide cómo enseñar conviene recordar que el proceso de aprendizaje es una construcción personal de cada estudiante que éste realiza con la ayuda de los mediadores (docentes o compañeros con más desarrollo y/o preparación); al ser una construcción personal, cada proceso puede ser distinto y consecuentemente las ayudas que precisa cada uno se concretarán según sus necesidades, capacidades e intereses. Ello nos plantea las conveniencia de tener presente los postulados del constructivismo y del aprendizaje significativo para responder a la diversidad. Así mismo, puede resultar de utilidad tener en cuenta una serie de criterios que orienten en el diseño de la metodología de enseñanza y aprendizaje debatida y consensuada en el PCC. Algunos de estos criterios pueden ser los siguientes: A) Promover, en todo el alumnado y desde los primeros cursos, la autonomía personal y social para desarrollar un trabajo autónomo en el aprendizaje y solidario con los demás. B) Combinar variados modelos de enseñanza/aprendizaje (sociales, personales, cognitivos, etc.), así como distintos lenguajes (verbal, icónico, plástico, etc.) y diferentes soportes de la comunicación (audiovisual, digital, gráfico, oral, escrito) con el fin de responder a las diferentes necesidades, capacidades y estilos de aprendizaje. C) Planificar una amplia serie de actividades didácticas en las que los docentes puedan observar cómo realizan el proceso de aprendizaje cada uno de sus alumnos y así poder adaptar las ayudas pedagógicas necesarias. D) Diseñar y organizar talleres y proyectos, así como resolución de problemas, comprobación de hipótesis y búsqueda de alternativas que permitan organizar los contenidos en torno 
a macro-actividades y responder a los diversos intereses, expectativas y capacidades. E) Aprovechar todo lo que ofrece el entorno "explotando" el significado que pueda tener para el alumnado del centro. F) Habituar al alumnado a hacerse guías de trabajo y estudio y cumplirlas, así como planes y actividades de autoevaluación y coevaluación para controlar el propio progreso y memorias de recapitulación sobre el proceso seguido; ello permitirá autorregular el aprendizaje por parte del propio alumno, de forma individual o en colaboración. En resumen, se trata de reconocer la necesidad de compaginar variados modelos, métodos y estrategias que, mediante sistemas de personalización, enseñanza individualizada y de trabajo cooperativo, se revelan como especialmente apropiados para una enseñanza que, para ser eficaz, no necesita clasificar segregando al alumnado . Para más información, entre otros autores, puede consultarse los siguientes: Ainscow, (2001 a), Ainscow y otros (2001), Blanchard y Muzás (2005), Echeita y Martín (1990), Mauri y Onrubia (1994), Monereo y otros (2000), Muñoz y Maruny (1993), Oliver (2003) y Pujolás (2001).

En séptimo lugar, en relación a la toma de decisiones acerca de la evaluación, cabe plantearse algunas cuestiones primordiales tales como: ¿qué se pretende? ¿qué es lo realmente importante? ¿qué consecuencias tendrá? ... Es conveniente, por tanto, establecer desde el centro criterios de evaluación, de información de resultados y de promoción. Pero, justamente, éstos serán diferentes según la respuesta a los anteriores interrogantes. La evaluación, desde un modelo inclusivo, pretende conocer de modo preciso cómo aprende cada alumno y alumna, cómo están adquiriendo las competencias y cómo desarrollan todas sus capacidades, para ayudar a todos a superar sus dificultades en el aprendizaje y en la participación, en definitiva, a aprender mejor; las decisiones que se deriven de la evaluación, en ningún caso se dirigirán a categorizar al alumnado y sí a fomentar el apoyo psicopedagógico necesario. Respecto a los criterios de evaluación, conviene tener en cuenta tres cuestiones básicas: qué, cuándo y cómo evaluar. En cuanto a qué evaluar, y en coherencia con este modelo, convendría comprobar en qué grado, cada estudiante, está logrando el desarrollo de capacidades y competencias propuestas en los objetivos generales de etapa y áreas. Y ello sin olvidar la referencia del propio sujeto que nos permitirá averiguar su avance, aprendizaje y desarrollo para, en el caso de que éstos no fueran idóneos, promoverlos movilizando todos los recursos disponibles de modo inmediato. En relación a cuándo evaluar, cabe señalar tres tipos de evaluación en función de situaciones clave: inicial, continua y final. La primera, con carácter diagnóstico, se realiza al comienzo de los procesos de enseñanza y aprendizaje con el fin de detectar la situación inicial de cada alumno/a. Ello va a permitir adaptar el diseño general a las diferentes capacidades, intereses, ideas previas, actitudes, etc., de cada uno. Con esta información sobre cada estudiante, estamos en condiciones de incluir nuevos contenidos o actividades, matizar alguno de ellos, darles otro enfoque, utilizar determinados recursos o quizás suprimir alguna parte. Como se ve, es un requisito muy importante para ajustar el currículo a las diferentes necesidades del alumnado. La evaluación continua, de carácter formativo, permite conocer al momento cómo está aprendiendo cada alumno y, en su caso, qué dificultades de aprendizaje experimenta. Ello facilita reorientar el proceso de enseñanza-aprendizaje y, cuando sea necesario, proporcionar la ayuda pedagógica adecuada antes de que se produzcan lagunas o errores en los aprendizajes que aboquen al fracaso escolar. La evaluación final, con carácter sumativo, acredita qué aprendizajes han conseguido al término del proceso. A este propósito, conviene analizar estos resultados en términos de con- 
traste con los objetivos planteados inicialmente y establecer qué posición ha alcanzado cada estudiante en relación a ellos. Como se desprende de lo anterior, la práctica adecuada con todo el alumnado de la evaluación diagnóstica y formativa son garantía de un aprendizaje idóneo y se revelan como claves para conseguir un efecto de prevención del fracaso escolar. Para decidir cómo evaluar, conviene tener presente la función que se le atribuye y la finalidad de la evaluación en cada momento del proceso; en coherencia con ello, se aplicarán los instrumentos precisos tales como observación sistemática y continuada del trabajo y logros de cada estudiante, pruebas escritas de distinta naturaleza, pruebas orales, entrevistas, cuestionarios, etc. Sólo de esta forma aseguraremos la evaluación integral de todas las competencias, en todo el alumnado y en todos los momentos del proceso. En cuanto a los criterios de información de resultados habrá que decidir qué información se va a proporcionar, si será detallada o global, con qué periodicidad, qué se pretende con ella, a quién se ofrecerá, cómo será la comunicación y lenguaje empleado, etc. Al hacerlo no hay que olvidar que sus efectos pueden representar un fuerte impacto en el alumnado y sus familias, así como servir de referente para otros docentes; especialmente cabe mencionar su influencia en la creación de expectativas de éxito o fracaso en cada sujeto, sus familias y maestros y la importancia que las mismas tienen en la motivación para el aprendizaje. A este respecto, una opción que parece adecuada es la "evaluación descriptiva" (Casanova, 1999) mediante la que se explicitan los resultados de modo sintético incluyendo también en detalle los logros y dificultades significativos que va experimentando cada estudiante; de este modo también se facilita la evaluación formativa, la coevaluación y la autoevaluación. En consecuencia, habrá que cuidar esta información para que, en cualquier caso, represente una oportunidad más para promover el aprendizaje y la participación. En relación a los criterios de promoción, conviene establecerlos teniendo muy presente respecto a cada alumn@, además de las competencias curriculares alcanzadas, sus características personales, sociales, intelectuales, etc., así como las de su posible grupo-aula de referencia. En cualquier caso, habrá que acordar: quién debe tomar las decisiones sobre la promoción del alumnado; qué aspectos concretos deberán tomarse en consideración para promocionar a otro ciclo; a quién conviene consultar; y cómo se van a tener en cuenta las opiniones de los demás implicados en el proceso de toma de decisiones (orientadores, profesorado, padres, propio sujeto).

En octavo lugar, habrá de organizarse un plan para atender a la diversidad en previsión de que el diseño común resulte insuficiente o parcialmente inadecuado para que, algunos alumnos y alumnas, alcancen su máximo aprendizaje y participación. Esa previsión implica, entre otras soluciones, organizar variadas modalidades de apoyo. Hacerlo desde un modelo inclusivo como el propuesto significa interpretar los apoyos como todas aquellas "actividades que aumentan la capacidad de un centro para responder a la diversidad de su alumnado. Todas las modalidades de apoyo se reúnen dentro de un único marco y se visualizan desde la perspectiva de los alumnos y su desarrollo, en vez de desde la perspectiva de la escuela o de las estructuras de la administración educativa" (Durán y otros, 2002: 60). En cualquier caso, parece conveniente tener en cuenta algunas consideraciones (Booth y Ainscow, 2000): A) Utilizar las prácticas de evaluación y apoyo psicopedagógico para reducir las barreras al aprendizaje y la participación del alumnado en lugar de para categorizarlo como con necesidades educativas especiales; en este sentido, destacar la conveniencia de que los informes de evaluación psicopedagógica se centren en identifi- 
car sus "puntos fuertes", sus posibilidades de aprendizaje y desarrollo más que sus deficiencias o limitaciones así como en la propuesta de cambios en las estrategias de enseñanza y aprendizaje para aumentar y mejorar la participación de aquellos en el currículo ordinario y para favorecer la relación con otros estudiantes; igualmente conviene que las Adaptaciones Curriculares Individuales $(\mathrm{ACl})$ se centren en proporcionar el acceso y apoyar la participación dentro del currículo común así como que sirvan para mejorar las estrategias de enseñanza y aprendizaje para todos los alumnos. B) Coordinar todas las posibles formas de apoyo, interpretarlas como responsabilidad de todo el profesorado y considerarlas como parte de un plan general de mejora de la enseñanza para atender la diversidad en todo el centro; se fomentarán las de carácter ordinario (afectan al 100\% del alumnado) frente a las específicas, utilizando éstas únicamente cuando aquellas no han funcionado; así mismo, no hay que olvidar que los apoyos, además de dirigirse a la prevención y tratamiento de las dificultades de aprendizaje, han de buscar aumentar la autonomía e independencia de los estudiantes. De modo específico, en relación a los alumnos cuya primera lengua no es el castellano, habrá de cuidarse de coordinar el apoyo para el aprendizaje del idioma con el resto de apoyos pedagógicos que precise cada caso; es probable que el cambio de país y cultura conlleven efectos que se conviertan en barreras para el aprendizaje y la participación, variables según los alumnos. Asimismo, cabe plantearse la necesidad de coordinar el apoyo pedagógico con el psicológico y emocional que puedan requerir alumnos con dificultades emocionales y de conducta $\mathrm{O}$, en general, para fomentar el bienestar de todo el alumnado y poder afrontar posibles situaciones de descontento, indisciplina, conflictos y conductas disruptivas.

\subsection{PROGRAMACIÓN DE AULA}

Programar el trabajo mediante las correspondientes unidades didácticas implica un proceso de reflexión y de toma de decisiones acerca de cómo se va a promover la consecución de aprendizajes concretos de todos los integrantes del grupo-aula al que se dirige, para que consigan los objetivos; programar consiste en "diseñar la experiencia educativa que vamos a ofrecer a nuestros alumnos" (Blanchard y Muzás, 2005: 59). Es importante que, en el proceso de su elaboración y correspondientes revisiones, se involucre activamente y con plena responsabilidad el profesorado de apoyo con el fin de que todo el alumnado consiga su máximo aprendizaje y participación. Para que realmente la programación resulte un instrumento de respuesta a la diversidad de todos conviene tener presente algunas consideraciones.

En primer lugar, dada la máxima concreción que representa este nivel de planificación curricular, ha de hacerse para el grupo-aula real teniendo en cuenta la especificidad del grupo como colectivo y las particularidades de cada estudiante, sus potenciales y limitaciones, así como sus intereses y necesidades educativas. Esto exige disponer de una información completa, válida y fiable acerca del grupo y sus integrantes que incluya, además de lo anteriormente mencionado, sus conocimientos y experiencias adquiridos y vividos fuera de la escuela. Tal información permite dar al tema elegido la orientación adecuada para todos, prever posibles dificultades o necesidades de ampliación, refuerzo, apoyo o modificación, hacer los oportunos ajustes y extraer claves para atender a cada estudiante. Para dar esta orientación general al tema, lógicamente, también habrá de tenerse en cuenta el correspondiente Proyecto Curricular que nos permitirá, entre otras cosas, identificar el nivel de 
competencia curricular alcanzado por cada uno de los estudiantes en los aprendizajes realizados y programar el trabajo para que todos sigan aprendiendo aprovechando todo el tiempo disponible.

En segundo lugar, conviene tener presente una serie de criterios en la formulación de competencias, objetivos y contenidos; algunos de ellos pueden ser los siguientes: A) Considerar el desarrollo de todo tipo de capacidades (cognitivas, sociales, afectivas, motoras, etc.) en la propuesta de objetivos y de desarrollo de competencias; esto con el fin de que no resulten sobredimensionadas las de naturaleza cognitiva frente a las demás y ello produzca desequilibrios en la formación integral de todos, así como para que no obre en perjuicio de aquellos alumnos cuyas capacidades tengan poco que ver con las requeridas por la vida académica convencional. B) Seleccionar los contenidos para desarrollar las competencias y alcanzar los objetivos de tal modo que resulten equilibrados los de naturaleza conceptual, procedimental y actitudinal. C) No olvidar que el grado de consecución de objetivos y contenidos así como el nivel de desarrollo de competencias puede variar en los diferentes alumnos y alumnas. D) Intentar que los objetivos y contenidos propuestos sean apropiados para todos los alumnos y alumnas del aula. E) Definir con claridad y precisión los objetivos y contenidos mínimos de cada unidad didáctica para intentar que todos los consigan. F) Incluir en la programación de la unidad didáctica, además de objetivos y contenidos mínimos, otros de carácter optativo y libre; y ello con la intención de que puedan elegir entre variadas opciones, así como para dar cabida a posibles iniciativas, intereses y deseos de diferentes alumnos, y que de este modo puedan resultar un elemento de motivación para el trabajo y el esfuerzo sostenidos.

En tercer lugar, en relación a la metodología, actividades de enseñanza-aprendizaje y evaluación de cada unidad didáctica, cabe plantearse lo siguiente: A) Crear en el grupo-aula un ambiente de colaboración para que se ayuden mutuamente, así como preparar y aplicar técnicas grupales y de trabajo cooperativo. B) Fomentar el esfuerzo personal, la responsabilidad de cada alumno y la originalidad mediante el trabajo autónomo o independiente, el estudio personal y el estímulo de la creatividad. C) Diseñar actividades para averiguar que capacidades, habilidades, experiencias y conocimientos previos tienen los componentes del grupo-aula. D) Planificar actividades de enseñanza-aprendizaje y el uso de recursos múltiples y variados, para atender a los distintos intereses, estilos cognitivos y de aprendizaje de todos los alumnos. E) Proponer actividades abiertas y con distinto grado de dificultad para que cada alumno las realice al máximo de sus posibilidades y según sus intereses. F) Planificar los tiempos de realización de tareas en el aula teniendo en cuenta las diferencias de ritmo entre los alumnos. G) Organizar las actividades de clase para facilitar la realización simultánea de distintas formas de trabajo: de modo autónomo (individual y/o en equipo) y dirigido por el docente del aula; de esta forma, el docente puede distribuir su atención entre el alumnado del aula según necesidades de cada uno de ellos, atendiendo en todo caso al máximo desarrollo de la autonomía de todos. H) Intentar que, en el curso de las clases, todos progresen, avancen, experimenten éxitos; para ello, seguramente, el docente necesitará evaluar de modo continuo y, eso, le permitirá ajustar la enseñanza al aprendizaje de todos y cada uno. I) Diseñar actividades de apoyo y refuerzo al mismo tiempo que otras de ampliación y profundización en previsión de que haya alumnos que, en el desarrollo de cada tema, experimenten dificultades o bien haya otros cuyo ritmo de aprendizaje sea más rápido o sus intereses y conocimientos sean mayores que los de otros compañeros. 
J) Planificar la evaluación de tal modo que sirva para valorar el progresos de cada uno e los alumnos y alumnas mediante la aplicación de variados procedimientos, técnicas e instrumentos de evaluación.

\section{CONCLUSIÓN}

El currículo ordinario puede representar una excelente oportunidad de respuesta a la diversidad de todo el alumnado cuando se analiza la normativa legal para aplicarla con intención inclusiva y se diseñan, desde el centro, los distintos niveles de planificación en clave de diversidad; ello exige interpretar ésta como una característica inherente a todo ser humano. Es preciso de todo punto huir del hipotético "alumno medio" porque, además de que éste no existe, suponerlo puede acarrear efectos nefastos en cuanto a promover decisiones que tiendan más a convertirse en soluciones selectivas que de carácter inclusivo para todos aquellos que se considera que se alejan de la "media". Abordar esta tarea no es fácil y requiere una clara convicción en los postulados de la educación inclusiva por parte de todos o, al menos, de una amplia mayoría de docentes; además de un fuerte y sostenido compromiso con ese modelo educativo; asimismo requiere una formación inicial y permanente que permita su aplicación generalizada. Todo ello ha de suponer no sólo la utilización de medidas de naturaleza curricular. Se requiere, también, generar estructuras de funcionamiento que atañen a la organización del centro $Y$ que propicien la creación de contextos inclusivos; exige la aplicación de variados modelos de enseñanza-aprendizaje cuya combinación permitirá tanto adaptarse a los diferentes ritmos y estilos de aprendizaje como el desarrollo de diferentes competencias en todo el alumnado. Para hacer realidad este modelo de educación inclusiva es preciso destacar, especialmente, la importancia capital de la orientación, considerando ésta responsabilidad tanto de los orientadores como de todos los docentes.

\section{BIBLIOGRAFÍA}

AINSCOW, M. (2001 a). Desarrollo de escuelas inclusivas. Ideas, propuestas y experiencias para mejorar las instituciones escolares. Madrid: Narcea.

AINSCOW, M. (2001 b).Comprendiendo el Desarrollo de Escuelas Inclusivas. Disponible en: http://innovemos.unesco.cl/medios/Documentos/DocumentosConsulta/epd/Ainscow2001esp.doc.

AINSCOW, M., BERESFORD, J., HARRIS, A., HOPKINS, D. y WEST, M. (2001). Crear condiciones para la mejora del trabajo en el aula. Manual para la formación del profesorado. Madrid: Narcea.

ARNAIZ, P.(2003). Educación inclusiva: una escuela para todos. Málaga: Aljibe.

BLANCHARD, M. y MUZÁS, M. D. (2005). Propuestas metodológicas para profesores reflexivos. Cómo trabajar con la diversidad del aula. Madrid: Narcea.

BOOTH, T. y AINSCOW, M. (2000). The Index for Inclusion: developing learning and participation in schools. Bristol: Centre for Studies on Inclusive Education (CSIE). (Traducción y adaptación al español, 2002: Madrid: Consorcio Universitario para la Educación Inclusiva).

CARDONA, M.C. (2005). Creencias, percepciones y actitudes hacia la inclusión: una síntesis de la literatura de investigación. En C. JIMÉNEZ (Coord.). Pedagogía Diferencial. Diversidad y equidad. Madrid: Pearson educación, pp 239-266. 
CASANOVA, M.A. (1999). Educación para una sociedad plural. En Organización y Gestión Educativa. Revista del Forum Europeo de Administradores de la Educación. Escuela Española, 2, pp 3-7.

DíAZ ALLUÉ, M. T., CARBALLO, R., FERNÁNDEZ DÍAZ, M. J. y GARCÍA NIETO, N. (1997). Orientación en Educación Secundaria. Situación actual y prospectiva. Revista Investigación Educativa, 15 (2), pp 9-83.

DíAZ ALLUÉ, M. T. (1999). Orientación educativa y atención a la diversidad del alumnado de Educación Secundaria Obligatoria. Bordón, 51, (1), pp 115-124.

DURÁN, D. y otros (2002). Guía para la evaluación y mejora de la Educación Inclusiva. Desarrollando el aprendizaje y la participación en las escuelas. (Traducción revisada y adaptada de la obra de BOOTH y AINSCOW, 2000). Consorcio.educacioninclusiva@uam.es.

ECHEITA, G. Y MARTíN, E. (1990) Interacción social y aprendizaje. En Marchesi, Coll y Palacios. Desarrollo Psicológico y Educación III. Necesidades Educativas Especiales y aprendizaje escolar. Madrid: Alianza, 49-67.

ECHEITA, G. y VERDUGO, M.A. (Coords.) (2004). La Declaración de Salamanca sobre Necesidades Educativas Especiales 10 años después. Valoración y prospectiva. Salamanca: Universidad de Salamanca, INICO.

GAIRÍN, J. (2001). Una escuela para todos: Un reto social y educativo. En SIPÁN, A. (Coord.). Educar para la diversidad en el siglo XXI. Zaragoza: Mira Editores, pp 241-266.

GARCíA GARCíA, M. (2002). Atención a la diversidad en Educación Secundaria Obligatoria. EduPsikhé: Revista de Psicología y Psicopedagogía, 1 (2), pp 225-248.

GARCÍA GARCÍA, M. (2005). Educación Adaptativa y escuela inclusiva: una forma de atender las diferencias de todos los estudiantes. En C. JIMÉNEZ (Coord.). Pedagogía Diferencial. Diversidad y equidad. Madrid: Pearson educación, pp 3-31.

JIMÉNEZ TRENS, M. A. (2004). La Declaración de Salamanca sobre Necesidades Educativas Especiales 10 años después. Valoración y prospectiva desde La Rioja. En ECHEITA, G. Y VERDUGO, M. A. La Declaración de Salamanca sobre Necesidades Educativas Especiales 10 años después. Valoración y prospectiva. INICO/ Universidad de Salamanca (pp149-159).

LEY ORGÁNICA 2/2006, de 3 de mayo, de Educación. Boletín Oficial del Estado, 4 de mayo.

MONEREO, C. (Coord.), CASTELLO, M., BASSOLS, M. y MIQUEL, E. (2000). Instantáneas. Proyectos para atender la diversidad educativa. Madrid: Celeste.

MAURI, T. y ONRUBIA, J. (1994) Decisiones sobre metodología didáctica. Cuadernos de Pedagogía, 223, pp 39-44.

MUÑOZ, E. y MARUNY, L. (1993) Respuestas escolares. Cuadernos de Pedagogía, 212, pp 11-14.

OLIVER, M. C. (2003) Estrategias didácticas y organizativas ante la diversidad. Dilemas del profesorado. Barcelona: OCTAEDRO-EUB.

PARRILLA, A. (2002). Acerca del origen y sentido de la educación inclusiva. Revista de Educación., 327, pp 11-29.

PARRILLA, A. y GALLEGO, C. (1999). Cómo pueden los profesores ayudarse entre sí: el desafío del trabajo compartido. Revista de Educación Especial, 25, pp. 55-69. 
PUJOLÀS, P. (2001). Atención a la diversidad y aprendizaje cooperativo en la educación obligatoria. Archidona: Aljibe.

R.D. 1513/2006, de 7 de diciembre, por el que se establecen las enseñanzas mínimas de la Educación Primaria. BOE, 8 de diciembre de 2006.

RIART VENDRELL, J. (Coord.) (2007). Manual de Tutoría y Orientación en la diversidad. Madrid: Pirámide.

SALEH, L. (2004). Desde Torremolinos a Salamanca y más allá. Un tributo a España. En ECHEITA, G. y VERDUGO, M.A. (Coords.) (2004). La Declaración de Salamanca sobre Necesidades Educativas Especiales 10 años después. Valoración y prospectiva. Salamanca: Universidad de Salamanca. INICO (pp 23-33).

SOLER, M. (1999). Diversidad y currículo. Trabajadores de la Enseñanza, 201, marzo. CCOO, pp 7-9.

SUSINOS, T. (2002). Un recorrido por la inclusión educativa española. Investigaciones y experiencias más recientes. Revista de Educación, 327, pp 4968.

SUSINOS, T. (2005). ¿De qué hablamos cuándo hablamos de inclusión educativa? Escuela Española, año V, 13, pp 4-6.

UNESCO/MEC (1994). Declaración de Salamanca y Marco de Acción para las Necesidades Educativas Especiales. Aprobada por la CONFERENCIA MUNDIAL SOBRE NECESIDADES EDUCATIVAS ESPECIALES: ACCESO Y CALIDAD. Salamanca, 7-10 de junio de 1994. Disponible en: http://www.unesco.org/education/nfsunesco/pdf/SALAMA. 